\title{
Efficient Priority Access to the Shared Commercial Radio with Offloading for Public Safety in LTE Heterogeneous Networks
}

\author{
Chafika Tata and Michel Kadoch \\ Department of Electrical Engineering, École de Technologie Supérieure, 1100 Rue Notre-Dame Ouest, \\ Montreal, QC, Canada H3C $1 \mathrm{~K} 3$ \\ Correspondence should be addressed to Chafika Tata; chafika.tata.1@ens.etsmtl.ca
}

Received 4 June 2014; Accepted 30 August 2014; Published 12 October 2014

Academic Editor: Lixin Gao

Copyright (C) 2014 C. Tata and M. Kadoch. This is an open access article distributed under the Creative Commons Attribution License, which permits unrestricted use, distribution, and reproduction in any medium, provided the original work is properly cited.

\begin{abstract}
This paper presents the algorithm Courteous Priority Access to the shared commercial radio with offloading (CPAwO), for public safety network (PSN) over LTE heterogeneous networks (HetNets). We propose a solution for prioritization of PSN users with access to the commercial radio network resources. Our model offers additional radio resources to the PSN. Furthermore, it ensures a certain priority for commercial users by assigning quantities of additional radio resources through the courteous scheme. This allows delaying preemption and blocking bearers when the radio resources are limited. The other part of CPAwO model is to apply the principle of offloading in order to reduce the impact of the macrocell congestion. This technique is to switch the new bearers arriving at the LTE macrocells to small cells, in order to decrease the number of the blocked and interrupted bearers in the network. The results of the simulation showed that the allocation of radio resources via the courteous mechanism with offloading of new bearers to small cells reduces the rate of blocking bearers and delays the preemption of active bearers present in the LTE HetNets. It also reduces the cost of the end-to-end communications, thanks to the reallocation of free frequencies.
\end{abstract}

\section{Introduction}

Wireless operators are facing a growing demand for communication broadband services. On the one hand, the satisfaction of the users is very important for the communication companies, while on the other hand, the lack of bandwidth and radio resources prevents wireless operators to meet all clients' requests. Long term evolution (LTE) over heterogeneous networks (HetNets) [1,2] is a pertinent solution to consider. The LTE resources have been used to improve the performance of public safety network (PSN) [3]. Indeed, the frequency band of $700 \mathrm{MHz}$ was dedicated to it in North America [4]. However, the increasing demand in terms of radio resources reduces the available PSN resources to ensure a good QoS, especially during times of crises. Therefore, sharing the commercial frequency band with the PSN network became an important solution addressed by several authors [4-7].

In this study a new approach for Courteous Priority Access to the shared commercial radio with offloading
(CPAwO) for the PSN over LTE HetNets networks is presented. The main objective of this solution is to ensure prioritization of PS users to access the shared commercial radio network resources. Our system as well as offering supplementary radio resources for the PS network ensures a certain priority for commercial users by assigning additional resources quantities through the courteous process. This is possible only if the QoS of the PS traffic is at an acceptable level. This approach enables delaying preemption and blocking of bearers during congestion. However, courteous algorithm may not be applied when the resources are exhausted in the LTE macrocell. For this purpose, another aspect of our solution is modeled to use unlicensed networks, such as WiFi, WMN, or ad hoc and to offload the LTE users (EU) of the congested macrocell. This has to be entirely transparent to the end. Hence, the EU uses the unlicensed network to exchange data. This is Device-to-Device (D2D) communications $[8,9]$. Our approach delays the preemption of bearers in the LTE HetNets network and reduces packet loss. In fact, new bearers will be redirected to the WMN 
instead of preempting the active bearers to release the needed radio resources. Offloading scheme is applied as long as the unlicensed network is not congested. Otherwise, the preemption must be applied on the lower priority active bearers.

Different radio resource allocation constraints models are built for managing the frequency resources quantity allocated to each traffic type, in order to guarantee a good quality of service for all bearers in the network. Courteous bandwidth allocation constraints model for frequencies (CAMF) is used to design a new frequency allocation model with constraints in terms of radio resources based on the courteous bandwidth allocation constraints (CAM) model [10]. CAM is a bandwidth allocation constraints model built especially, but not exclusively, for the MPLS network. It focuses on improving the allocation of resources for the classes of lower priority traffics. The CAM enhances the traffic allocation in the network compared to the two approaches, maximum bandwidth constraints allocation model (MAM) [11] and Russian dolls bandwidth constraints allocation model (RDM) [12]. In this work, we adapt the CAM model for the frequencies allocation scheme. Finally, three models have been developed, namely, the basic model CAMF (CAM for frequencies), the generalized CAMF model G-CAMF, and RUS-CAMF model (radio usage situation based courteous allocation constraints model for frequencies). These models are detailed below.

Note that RUS-CAMF and G-CAMF may be used by CPAwO solution; besides, CAMF is used by our previous solution CPA algorithm [5]. CPA (Courteous Priority Access algorithm) is one of our contributions published in [5]. CPA aims at enhancing the radio resources allocation for the Commercial and public safety networks. The main objective of CPA is to allow the PS network to access the shared commercial radio over LTE with priority, without penalizing commercial users. The PS network may give an amount of its resources if the $\mathrm{CN}$ one suffers from a high rate of packet loss. When comparing with the solution given by [4], the simulation results show that CPA algorithm reduces the number of the commercial blocked bearers, delays the $\mathrm{CN}$ blocking mechanism, increases the number of commercial active bearers, and keeps an acceptable level of PS blocking bearers. Note that when resources are limited for PS network, it may not yield any part of its dedicated resources. At this time, no more courteous allocation is done and a preemption will be invoked.

This paper is organized as follows. Section 2 presents the related work. In Section 3 the CPAwO algorithm is modeled. Section 4 illustrates the different radio resource allocation constraints models. Section 5 summarizes the most important simulation results and their interpretation. Section 6 concludes this paper. Finally, Section 7 presents the future works relevant to this study.

\section{Related Work}

Several studies in the literature deal with the radio resources allocation issue in LTE network [13-15]. The objective is to provide efficient schemes to manage the radio resources in the LTE and LTE HetNets networks. Article [13] offers a new solution to the radio resources allocation through a novel radio admission control (RAC) mechanism. RAC proposes to divide bearers into three groups, namely, the first group which includes the bearers vulnerable to preemption, the second group including the nonpreempting bearers which can interrupt bearers from the first group, and the third one, which contains the nonpreempting bearers that cannot interrupt others bearers. Furthermore, bearers in the same group may share the same amount of radio resources. Therefore, once the second group resources are limited, its bearers can interrupt others bearers from the first group. Authors in [14] propose a control admission mechanism combined with a congestion control scheme as a solution to reduce the blocking and the loss calls probability in the system. In [15], high priority bearers interrupt lower priority active bearers until getting the necessary resources to reach the minimum required QoS level. This may decrease the number of bearer's preemption in the network. Some other studies address the problem of public safety user's access to the shared commercial radio over the LTE and the LTE HetNets. The two papers $[4,5]$ develop solutions to provide a priority access to the shared commercial radio for the PS clients over the LTE network. In the first paper, PS users may use only a part of the shared radio even if no commercial user requests the radio resources. Besides, commercial users can use all the radio bands but can be interrupted if PS bands are requested by PS clients. Therefore, commercial bearers may suffer from high level of preemption. The second paper offers additional resources to the commercial users from the PS radio, while the QoS level of the PS traffic remains acceptable, which delays the preemption of commercial bearers and reduces call loss.

Managing radio resources is efficient to enhance the radio resources allocation in the LTE network when resources are available. But once all resources are allocated, and no preemptable bearer is active in the system, the level of QoS of different traffics will decrease. To address this issue, the bearers offloading mechanism from the macrocell to the small cells can be applied. The study [16] discusses the offloading of bearers from an LTE macrocell to WiFi network. This approach considers the case where the small cells position discovery is performed by the cellular network, in order to optimise the number of D2D connections. The authors demonstrate that their model can provide significant improvement in capacity and energy consumption. Other solutions are presented in to deal with the enhancement of D2D communications [17-19].

\section{Courteous Priority Access to the Shared Commercial Radio with Macrocell Offloading for PSN over LTE HetNets}

In this section we detail the PS network Courteous Priority Access with offloading approach to the commercial radio over the LTE HetNets, namely, CPAwO. We offer a solution for Commercial Radio Access with prioritization for the PSN. Our scheme gives additional radio resources to the PS 
network. Moreover, it keeps a certain priority for commercial users by assigning an amount of residual PS radio resources through the process of courtesy. This is possible only if the QoS of the PS traffic is acceptable. This approach delays preemption and blocking bearer's process when the radio resources are limited for the commercial network. The other step of the CPAwO approach is offloading the macrocells in order to reduce the congestion within them. This technique consists of switching the new bearers arriving at the congested LTE macrocells to small cells, such as WiFi, WMN, and ad hoc cells, a totally transparent operation to users which is able to reduce the number of blocked bearers and interrupted ones in the whole network.

Following is the detailed CPAwO scheme, including the bearers classification which is a priority calculation model.

\subsection{Bearers Classification and Priority Calculation. As we} mentioned before, all bearers' types may share the available radio resources in the LTE HetNets. However, all bearers do not have the same priorities in terms of access to radio resources and not all bearers can access these resources when they are limited. Therefore, it will be relevant to classify the bearers according to their priority level. In this section we discuss the classification and priority calculation of different bearers in the LTE HetNets. Indeed, in this study, we classify bearers of commercial network together with bearers of PS network. Moreover, the bearers belonging to the same network, PS or $\mathrm{CN}$, are classified too.

The classification of bearers, according to their priority, is very important for the ARP mechanism during the admission of new bearers or the modification of active bearers in the LTE HetNets and also during the preemption of some bearers to release some radio resources in favour of higher priority bearers.

The priority calculation is performed when a new bearer establishment is requested. Three elements affect the calculation of the priority of a bearer PS or CN, namely, the usage status represented by $\alpha$, which specify if the bearer establishment is relevant to an emergency or nonemergency situation, the ARP value related to this bearer, noted $\vartheta(\mathrm{Br})$, and the QCI value of the bearer, noted QCI(Br). Authors in [4] give more details about these three elements.

Thus, we propose the following formula for the calculation of the priority coefficient of a $\mathrm{Br}$ bearer:

$$
\Phi(\mathrm{Br})=\alpha * \vartheta(\mathrm{Br}) *(\beta+\mathrm{QCI}(\mathrm{Br}))
$$

where $\Phi(\mathrm{Br})$ is the bearer priority coefficient, $\alpha$ is the usage status $=\{0.1$, if emergency; 3 , otherwise $\}, \vartheta(B r)$ is ARP value relevant to the bearer, and $\mathrm{QCI}(\mathrm{Br})$ is $\mathrm{QoS}$ class identifier, $\beta=$ $\{\max (\vartheta(\mathrm{Br}))=8$, if bearer_type $=\mathrm{CN} ; 0$, Otherwise $\}$.

Note that as the value of $\Phi(\mathrm{Br})$ is small, the priority of the bearer increases. Furthermore, in our work we consider that all emergency PS packets have higher priority than emergency $\mathrm{CN}$ ones to access the PS dedicated spectrum band belonging to the shared commercial radio. By the same way, the whole nonemergent PS traffic is prioritized over all nonemergent $\mathrm{CN}$ ones on access to the shared spectrum band. This is made possible by the value of $\beta$. $\beta$ ensures that the lower priority bearer of PS will have lower value for $\Phi$ than the high priority CN bearer. Moreover, emergency calls PS or $\mathrm{CN}$ must have higher priorities than the nonemergency one, so lowest coefficients $\Phi$. To address this issue, the usage status, namely $\alpha$, is considered for the computation of the priority coefficient of the bearer.

Remember that QCI values vary between 1 and 9 inclusive and ARP varies from 1 to 15 inclusive [4]. The priority calculation's numerical modeling is given below. The objective is to show that our approach allows prioritizing higher traffic priority over lower priority one, and PS traffic is compared to the $\mathrm{CN}$ traffic.

Formulas (2)-(10) show the coefficients of priority traffic on two different usage situations, including emergencies and a classic situation. The $\operatorname{Min}(i)$ and $\operatorname{Max}(i)$ notations mean, respectively, minimal value and maximal one for a variable $i$, with $i \in \mathbb{R}$. Also, we take $\mathrm{Br}_{\mathrm{PS}}$ as a PS bearer and $\mathrm{Br}_{\mathrm{CN}}$ as an $\mathrm{CN}$ bearer:

$$
\operatorname{Min}\left(\Phi_{\text {status }}(\mathrm{Br})\right) \leq \Phi_{\text {status }}(\mathrm{Br}) \leq \operatorname{Max}\left(\Phi_{\text {status }}(\mathrm{Br})\right) .
$$

If status = emergency then $\alpha=0.01$ and

$$
\begin{aligned}
& \operatorname{Min}\left(\Phi_{\text {emergency }}(\mathrm{Br})\right) \\
& =\operatorname{Min} \alpha * \operatorname{Min}\left(\vartheta\left(\mathrm{Br}_{\mathrm{PS}}\right)\right) * \operatorname{Min}\left(\mathrm{QCI}\left(\mathrm{Br}_{\mathrm{ps}}\right)\right) \\
& =0.01 * 1 * 1=0.01, \\
& \operatorname{Max}\left(\Phi_{\text {emergency }}(\mathrm{Br})\right) \\
& =\operatorname{Max}\left(\Phi_{\text {emergency }}\left(\mathrm{Br}_{\mathrm{CN}}\right)\right) \\
& =\alpha \operatorname{Max}\left(\vartheta\left(\mathrm{Br}_{\mathrm{CN}}\right)\right) *\left(\beta+\operatorname{Max}\left(\mathrm{QCI}\left(\mathrm{Br}_{\mathrm{CN}}\right)\right)\right) \\
& =0.01 * 15 *(8+9)=2.55, \\
& \quad 0.01 \leq \Phi_{\text {emergency }}(\mathrm{Br}) \leq 2.55 .
\end{aligned}
$$

Also, when we apply formula (1) and consider the ARP and QCI values of bearers, we get

$$
\begin{aligned}
& 0.01 \leq \Phi_{\text {emergency }}\left(\mathrm{Br}_{\mathrm{PS}}\right) \leq 0.72, \\
& 0.81 \leq \Phi_{\text {emergency }}\left(\mathrm{Br}_{\mathrm{CN}}\right) \leq 2.55
\end{aligned}
$$

If status $=$ nonemergency then $\alpha=3$ and

$$
\begin{aligned}
\operatorname{Min} & \left(\Phi_{\text {nonemergency }}(\mathrm{Br})\right) \\
= & \operatorname{Min}\left(\Phi_{\text {nonemergency }}\left(\mathrm{Br}_{\mathrm{PS}}\right)\right) \\
= & \alpha * \operatorname{Min}\left(\vartheta\left(\mathrm{Br}_{\mathrm{PS}}\right)\right) * \operatorname{Min}\left(\mathrm{QCI}\left(\mathrm{Br}_{\mathrm{PS}}\right)\right) \\
= & 3 * 1 * 1=3, \\
\operatorname{Max} & \left(\Phi_{\text {nonemergency }}(\mathrm{Br})\right) \\
= & \operatorname{Max}\left(\Phi_{\text {nonemergency }}\left(\mathrm{Br}_{\mathrm{CN}}\right)\right) \\
= & \alpha * \operatorname{Max}\left(\vartheta\left(\mathrm{Br}_{\mathrm{CN}}\right)\right) *\left(\beta+\operatorname{Max}\left(\mathrm{QCI}\left(\mathrm{Br}_{\mathrm{CN}}\right)\right)\right) \\
= & 3 * 15 *(8+9)=765, \\
3 \leq & \Phi_{\text {non-emergency }}\left(\mathrm{Br}_{i}\right) \leq 765, \quad i=\mathrm{PS} \text { or } \mathrm{CN} .
\end{aligned}
$$


Also

$$
\begin{gathered}
3 \leq \Phi_{\text {nonemergency }}\left(\mathrm{Br}_{\mathrm{PS}}\right) \leq 216, \\
243 \leq \Phi_{\text {nonemergency }}\left(\mathrm{Br}_{\mathrm{CN}}\right) \leq 765 .
\end{gathered}
$$

The numerical model proposed above for the calculation of bearers' coefficients of priority ensures that during transmissions belonging to the same communication status, namely, an emergency situation or nonemergency one, the PS traffic has priority over the $\mathrm{CN}$ traffic, see formulas (6) and (7) and formulas (9) and (10). Moreover, the model guarantees the prioritization of the emergency $\mathrm{CN}$ traffic over the nonemergency PS traffic, see formulas (7) and (9). Note that this model is based on the ARP and QCI values defined in the LTE standard as used in [4]. An eventual QCI and ARP values change do not affect our mechanism. Indeed, a suitable choice of the values of $\alpha$ and $\beta$ is possible so that the current formulas (1) and (2) are still valid.

Our classification model allows four principal types of bearers, namely, PS emergency bearers which deal with the PS communication during emergency situations, the PS nonemergency bearers that depend on the nonemergency PS transmissions, the $\mathrm{CN}$ emergency and the $\mathrm{CN}$ nonemergency bearers which are relevant to the urgent commercial communications, and the nonurgent commercial ones, respectively.

Authors in [4] give a classification solution by considering the PS emergency as the higher priority bearers group, followed by the PS nonemergency one. The third priority is given to the $\mathrm{CN}$ emergency and the last one is given to the $\mathrm{CN}$ nonemergency one. As we work on a shared commercial radio bands, we believe that only the PS emergency traffic has to be more prioritized than the $\mathrm{CN}$ emergency one, because on one hand, the PS traffic has already its dedicated radio band and on the other hand, during situation of crisis, the transmission of the $\mathrm{CN}$ emergency traffic is very important too. Therefore, our proposed classification scheme offers the highest priority to the PS emergency traffic, followed by the $\mathrm{CN}$ emergency traffic, after that, we give the third priority level to the PS nonemergency traffic and finally the $\mathrm{CN}$ nonemergency traffic.

\subsection{Implementation and Description of CPAwO Algorithm.} $\mathrm{CPAwO}$ algorithm is a generalization of CPA scheme [5]. The first contribution of $\mathrm{CPAwO}$ regarding $\mathrm{CPA}$ is the scalability of our solution. The generalization of CPA enhances the radio resources allocation with constraints for $n$ frequency group classes, with $\in \mathbb{N}$. CPAwO scheme is a flexible solution since it allows a multiple bearers gathering to construct a frequency class group (FCG). This is possible as the priority coefficients $\Phi$ of these bearers are within the same range values $[a, b], a$, and $b \in \mathbb{R}$. Moreover, this approach may be used, theoretically, to an infinite number of FCG groups.

The CPAwO algorithm is a radio resource allocation mechanism with constraints on the resources reserved for each group of bearers FCG. The quantity of the allocated frequency bands depends on the value of the priority of the FCG group. This principle is applied to ensure the availability of a portion of resources to high priority bearers as well as to guarantee the service to lower priority bearers. However,
CPAwO is not limited to the management of the radio resource allocation constraints but is also interested in the redistribution of unused resources, by courteous scheme, given to the disadvantaged classes. This is applicable under certain conditions called courteous conditions; namely,

(i) the resources assigned by courteous scheme must belong to the group of bearers of the adjacent high priority. Recipients of resources by courteous mechanism, must belong to adjacent groups of lower priority;

(ii) the rate of packet loss of high priority class must be acceptable and below the tolerable packet loss threshold;

(iii) the rate of packet loss of the lower priority class must be critical, therefore, exceeds the threshold of tolerable packet loss in order to benefit from the courtesy.

Certainly, underutilized resource reallocation improves the quality of service of bearers belonging to disadvantaged frequency group classes, since it can delay the preemption of the lower priority active bearers in the network and succeed even in delaying blocking bearers. Besides, more bearers will be admitted in the network, thanks to the resources availability.

Algorithms 1, 2, 3, and 4 detail the $\mathrm{CPAwO}$ algorithm and the functions associated with it.

In the initial state, all the resources are available and the rate of the allocated radio is null. These resources are allocated as far as a new establishment or modification of bearers request is formulated. For each request the required resources related to the establishment of the bearer is estimated, and the group FCG of the bearer and the status of its use, namely, crisis management or simple communication is determined. This information is mandatory for computing the coefficient of priority of the bearer $\Phi$ (formula (1)).

The classification of bearers within FCG groups is performed regarding the value of $\Phi$. Remember that bearers having coefficient values of the priority coefficient $\Phi$ included in the same interval $[a, b]$, where $a, b \in \mathbb{R}$, will be put in the same FCG. Defining FCG group membership is relevant for the allocation type constraints applied to the bearer of the GCAMF, which will be detailed later in this paper (formula (15) and Figure 2, generalized CAMF).

If resources are limited then upper adjacent group $\mathrm{FCG}_{i+1}$ will be requested for additional resources. Therefore, the algorithm will run the courteous scheme (Figure 3). If the conditions of courteous mechanism, cited above, are checked and unused resources can meet the demand of a new bearer, the unused or extra resources assigned to the group $\mathrm{FCG}_{i}$ will be allocated to the $\mathrm{FCG}_{i+1}$ group. This will be done temporary since the QoS of the $\mathrm{FCG}_{i}$ is still acceptable. Otherwise, the courteous resources allocation request will be rejected.

Certainly courteous resources allocation request rejection causes a deterioration of the QoS of the related FCG. Above all, the demand of additional resources by courteous scheme is performed only during congestion time. To address this issue, the $\mathrm{CAPwO}$ presents an appropriate solution to 


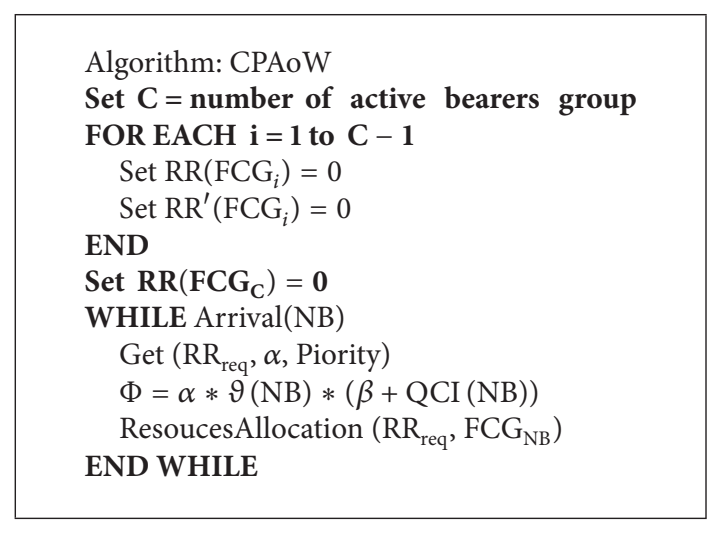

Algorithm 1: CPAwO algorithm.

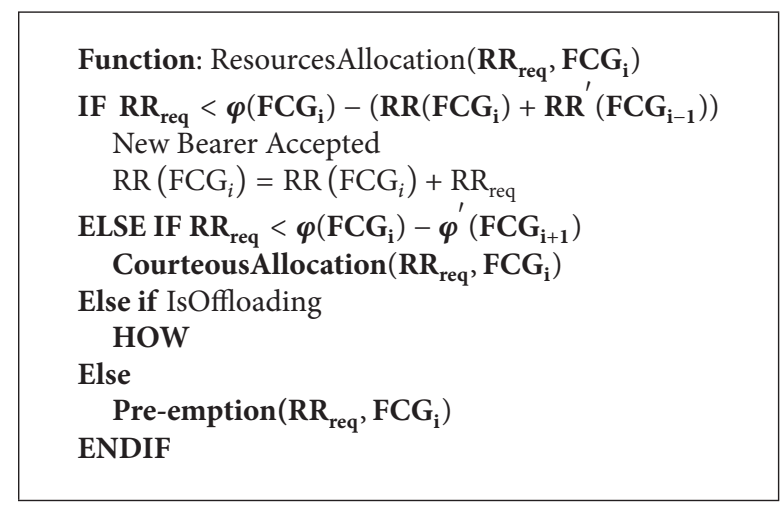

Algorithm 2: Resources allocation function.

improve the performance of disadvantaged traffic, namely, the use of the unlicensed frequencies in order to acquiesce the new bearer's requests, when the whole radio resources are allocated at the macrocell. A contribution is added to our mechanism for managing the radio resources allocation within the LTE HetNets.

$\gamma_{j}$ is the number of admitted bearers in the small cell $j$ of the macrocell LTE. Therefore, we note $\Omega$ as the gain in number of extra bearers admitted within the whole LTE HetNet network. Following is the calculation of $\Omega$ :

$$
\Omega=\sum_{j=1}^{n} \gamma_{j} .
$$

Small cells are subject to saturation. After the exhaustion of available resources within the unlicensed radio, no small cell will accept any additional LTE bearer. There we talk about a lack of radio resources. In such a case, the CPAwO interrupts low priority preemptable active bearers in the network. Preemption is done according to rules established by the LTE standard and applied in [4].

Indeed, during congestion time, and when resources are not sufficient to satisfy the bearer's requests because of the saturation of the macrocells in the LTE network, the CPAwO algorithm executes the handoff to the WLAN

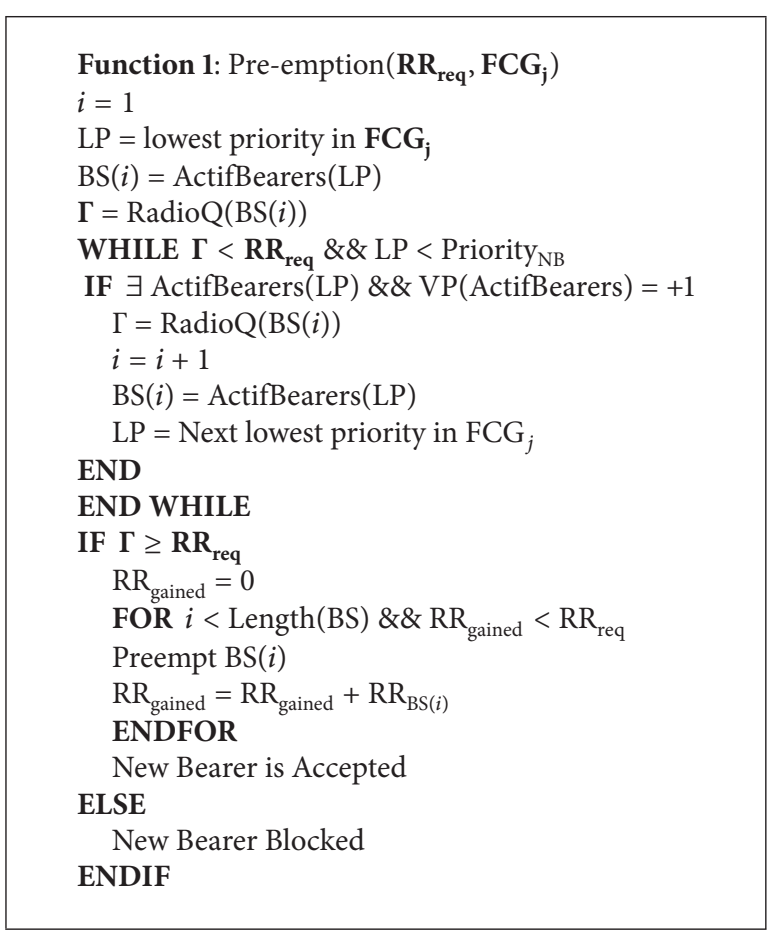

Algorithm 3: Preemption function.

(HOW) algorithm. HOW scheme can offer an additional radio resources to the PSN and $\mathrm{CN}$ networks. Furthermore, it may reduce the congestion degree by offloading the bearers from the macrocell to small cells inside the LTE HetNets network. Though, instead of rejecting bearers or interrupting others belonging to the lower priorities, "HOW" mechanism performs switching of new bearers to WLAN network, such as WMN, ad hoc, or WiFi. Note that HOW chooses to redirect a new bearer of high priority to small cells instead of preempting a lower priority active bearer. This approach ensures high level of stability in the LTE HetNets network.

The proposed solution focuses on two factors relevant to the improvement of the performance of QoS in LTE HetNets networks, namely, delaying the preemption of active bearers in the network by redirecting new bearers to small cells and increasing the number of active bearers in the whole network. Remember that the overall number of active bearers throughout the network is equal to the sum of active bearers in the macrocells and the small cells.

3.3. Data Offloading to Small Cells Algorithm. As mentioned below, the courteous algorithm may not be applied when resources are limited for the PS network. In such cases preemption is inevitable. Hence, the other aspect of our solution is to use unlicensed networks, such as WiFi, WMN, and ad hoc networks, to offload the LTE HetNets users (EUs). This should be entirely transparent to the EUs. Therefore, the EU uses the unlicensed network to exchange data rather than LTE frequency bands. This is the Device-to-Device (D2D) communication. This approach delays the preemption of bearers in the LTE HetNets network and reduces packet loss even more. In fact, new bearers will be redirected to 


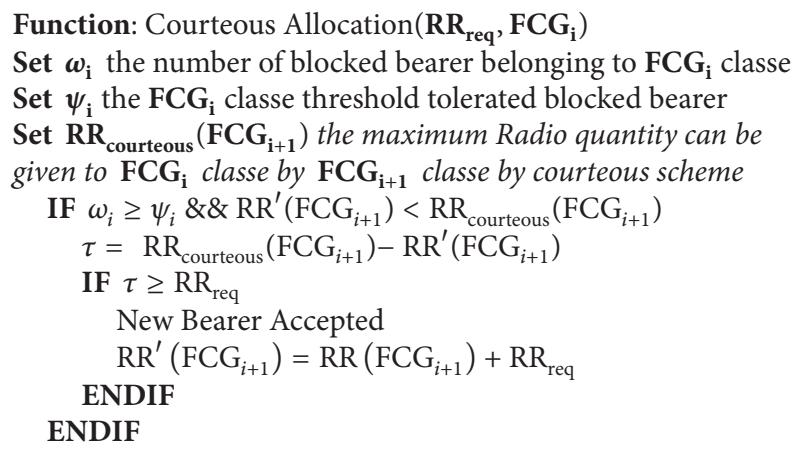

Algorithm 4: Courteous for frequencies function.

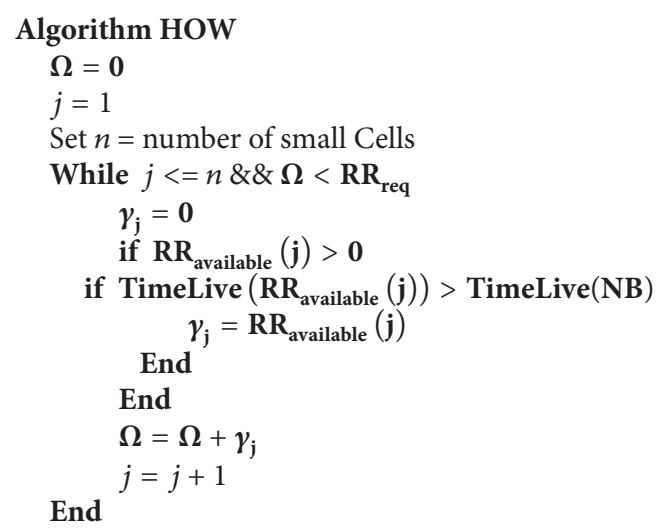

Algorithm 5: Algorithm handoff to WLAN (HOW).

the WMN instead of preempting the active bearers to release the needed radio resources. Offloading scheme is applied as long as the unlicensed network is not congested. Otherwise, the preemption must be applied on the lower priority active bearers.

The principle of the HOW algorithm scheme is to switch the new bearers to the small cells to the macrocell LTE HetNet network, during congestion times. A new bearer is redirected to small cells even if it has a higher priority. On the other hand, the low priority bearers remain active in the network and are still served by the macrocells, despite the arrival of higher priority bearers. The objective is to minimize the number of handoff in the network. However, this strategy should not affect the QoS of the high priority bearers, which will be admitted within the public frequencies. Note that offloading operation occurs only if the following conditions apply:

(1) resources must be exhausted within macros cells. This is represented by formula (23). This formula interprets the case where each reserved radio by FCG $_{i}$ must reach the maximum amount of reservable radio resources, where $i$ is the number of group classes of active frequencies in the network:

$$
\forall i \in C, \quad \mathrm{RR}\left(\mathrm{FCG}_{i}\right)=\varphi\left(\mathrm{FCG}_{i}\right) .
$$

(2) Radio resources have to be available in at least one small cell and should be sufficient enough to satisfy the request for the establishment of the arrived bearers (Algorithm 5).

(3) The duration time of the available resources in the cell must be greater than or equal to the duration time of the bearer to be established (Algorithm 5).

\section{Radio Resource Allocation Models}

CAMF is a new frequency allocation model with constraints in terms of radio resources, based on the CAM model [10]. CAM is a bandwidth allocation constraints model built especially, but not exclusively, for the MPLS network. It focuses on improving the allocation of resources for the classes of lower priority traffic. The CAM enhances the traffic allocation in the network with respect to the two approaches MAM [11] and RDM [12]. In this work, we adapt the CAM for the frequencies allocation. Three models have been developed, namely, the basic model CAMF (CAM for frequencies), the generalized model G-CAMF (generalized CAMF), and RUSCAMF model (radio usage status based courteous allocation constraints model for frequencies). These models are detailed below. "Defined of Used Variables" Section summarizes the different variables used in the two allocation models for frequencies, namely, the classical approach and the courteous one.

4.1. Classical Approach to Priority Access to the Shared Commercial Radio. Authors in [4] describe the access solution to the shared commercial frequency band for the PSN users. This approach, which we call classical approach in this paper, illustrates the sharing of commercial radio resources between the PS bearers and CN ones.

In this solution, $\mathrm{CN}$ bearers may use the whole shared radio bands when no PS bearer is requesting resources. Against, PS bearers may only use part of the shared bands 
even if no $\mathrm{CN}$ bearers request the resources. On the other hand, PS dedicated frequencies allocated to $\mathrm{CN}$ bearers are released as soon as a PS bearer requests them.

Many conditions apply for preemption of an active bearer $(A B)$ in the network by a new bearer (NB). Indeed, a lower priority bearer has no ability to interrupt another higher priority. In addition, a new bearer needs to have the attribute capacity to preemption (CP) set to yes, which we represent in this work by the positive value +1 . A bearer with a $\mathrm{CP}$ value set to no, which is equal to -1 , will not be able to interrupt the active bearers. In addition, the bearer which can be interrupted must be vulnerable to preemption. This value, expressed by VP, must be equal to yes. In our solution we attribute the positive value +1 to VP if the corresponding bearer is vulnerable to preemption, -1 otherwise.

Based on the preemption process described in [4], we define the following formulas:

$$
\begin{aligned}
& \text { Condition }_{1}=\left\{\operatorname{Pri}(\mathrm{NB})>\operatorname{Pri}\left(\mathrm{BA}_{\mathrm{vp}}\right),\right. \\
& \mathrm{CP}(\mathrm{NB})=+1 \text {, } \\
& \left.\operatorname{VP}\left(\mathrm{BA}_{\mathrm{vp}}\right)=+1\right\} \text {, } \\
& \text { Condition }_{2}=\left\{\operatorname{Pri}(\mathrm{NB}) \leq \operatorname{Pri}\left(\mathrm{BA}_{\mathrm{vp}}\right)\right. \text { or } \\
& \mathrm{CP}(\mathrm{NB})=-1 \text { or } \\
& \left.\operatorname{VP}\left(\mathrm{BA}_{\mathrm{vp}}\right)=-1\right\} \text {, }
\end{aligned}
$$

Preemption $\left(\mathrm{NB}, \mathrm{BA}_{\mathrm{vp}}\right)=\left\{\begin{array}{l}\text { True if Condition } \\ \text { False if Condition }\end{array}\right.$

Figure 1 shows the model of radio resource allocation based on the classical approach [4] (Figure 1(a)) and the adaptation of the CAM [10] for frequencies (CAMF) (Figure 1(b)). Note that CAMF is the model used by CPA as a constraints allocation scheme.

In the classical approach [4], the allocation of radio resources with constraints model can be represented by the three following joint forms.

(1) For $i=\mathrm{CN}$, and $j=\mathrm{PS}$

$$
\mathrm{RR}_{s}(i)+\mathrm{RR}_{s}(j) \leq \varphi_{s}(i),
$$

(2) with the constraint

$$
\sum_{x=\{\mathrm{CN}, \mathrm{PS}\}} \mathrm{RR}_{d}(x)+\mathrm{RR}_{s}(x) \leq \varphi_{\mathrm{Max}} .
$$

(3) Finally

$$
\sum_{x=\{\mathrm{CN}, \mathrm{PS}\}} \varphi_{d}(x)+\varphi_{s}(x) \geq \varphi_{\mathrm{Max}} .
$$

The classical approach of frequency allocation is modeled to share resources between PS and CN radio networks, so that a portion of the resources will be dedicated to the $\mathrm{CN}$, and another will be dedicated to the PS. In addition, a frequency band will be shared between the two networks. To do this, the following conditions apply.

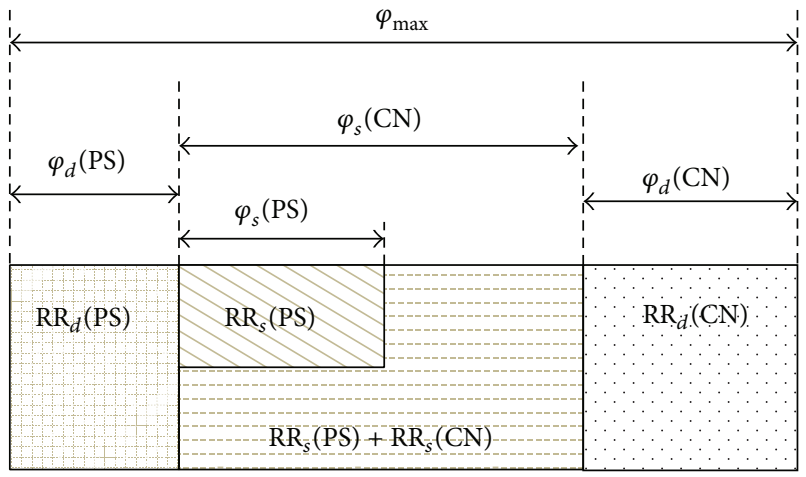

(a) Conventional ARP approach

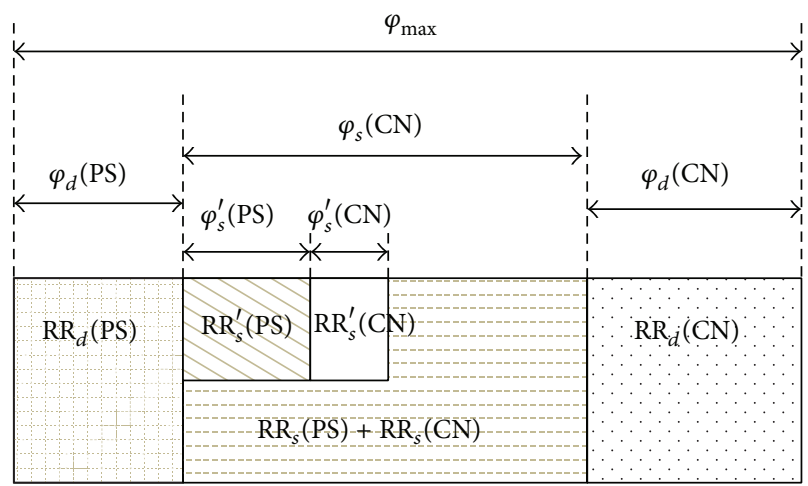

(b) CAMF approach

FIGURE 1: Courteous allocation model for frequencies.

(1) The shared frequency band may be allocated by the $\mathrm{PS}$ and $\mathrm{CN}$ bearers.

(2) PS bearers may allocate an amount of resource radio within the shared frequency band. But, the shared radio quantity allocated must not exceed the value $\varphi_{s}(\mathrm{PS})$. This represents the maximum threshold of PS shared radio allocation.

(3) The bearers of type $\mathrm{CN}$ may use a portion of the shared radio. This portion can allocate the total shared resources if there is no PS bearer which requests for the establishment of bearer. $\mathrm{CN}$ users release shared radio resources dedicated to PS as soon as they are claimed.

4.2. Courteous Allocation Model for Frequencies. For CAMF, the allocation of radio resources with courtesy is given as follows.

(1) For $i=\mathrm{CN}$, and $j=\mathrm{PS}$

$$
\mathrm{RR}_{s}(i)+\mathrm{RR}_{s}(j) \leq \varphi_{s}(i),
$$

(2) with the constraint

$$
\sum_{x=\{\mathrm{CN}, \mathrm{PS}\}} \mathrm{RR}_{d}(x)+\mathrm{RR}_{s}(x) \leq \varphi_{\mathrm{Max}} .
$$




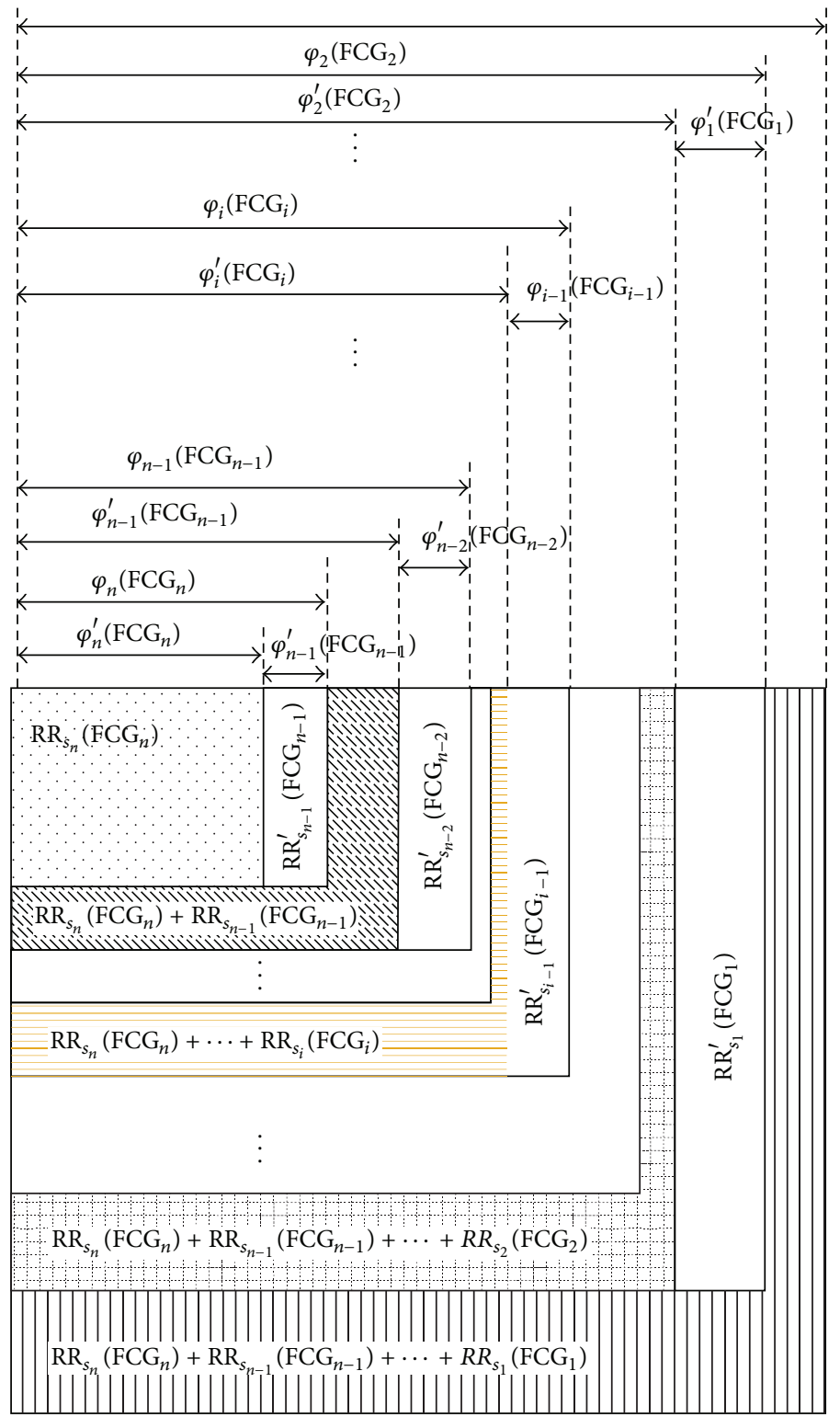

Figure 2: Generalized CAMF.

(3) For $i=\mathrm{CN}$, and $j=\mathrm{PS}$

$$
\begin{aligned}
& \operatorname{RR}_{s}(j)=\operatorname{RR}_{s}^{\prime}(j)+\operatorname{RR}_{s}^{\prime}(i), \\
& \operatorname{RR}_{s}^{\prime}(j) \leq R_{\text {th,s }}(j) .
\end{aligned}
$$

(4) Finally

$$
\sum_{x=\{\mathrm{CN}, \mathrm{PS}\}} \varphi_{d}(x)+\varphi_{s}(x) \geq \varphi_{\mathrm{Max}} .
$$

The CAMF model differs from the traditional approach by the way the shared commercial frequency band is allocated. Indeed, the portion of resource radio allocated to $\mathrm{CN}$ and dedicated to PS will not be released as soon as the new PS bearer call request. The PS network could waive its request of resources, where the packet loss rate is considerable within the $\mathrm{CN}$ network. This is possible only when the $\mathrm{CN}$ packet loss rate is greater than a threshold $\mathrm{TH}_{\mathrm{pk}_{\mathrm{lost}}}(\mathrm{CN})$. Furthermore, the level of quality of service of the PS network is acceptable. This is interpreted by a rate of allocation of resources by PS radio below the threshold of tolerance (formula (3) of the CAMF model) [20].

4.3. Generalized Courteous Constraints Allocation Model for Frequencies. Figure 2 shows the generalization of the CAMF model detailed below in this paper, for $\mathrm{FCG}_{n}$, with $n \in$ $\mathbb{N}$, considering the allocation constraints for each frequency class group $\left(\mathrm{FCG}_{k}\right), k \in\{1, C\}, C$ is the number of active $\mathrm{FCG}$ in the network. Note that all bearers belonging to the same FCG hold a coefficient of priority between $\mathrm{cp}_{1}$ and $\mathrm{cp}_{2}$, where $\mathrm{cp}_{1}$ is the coefficient of priority corresponding to the higher 


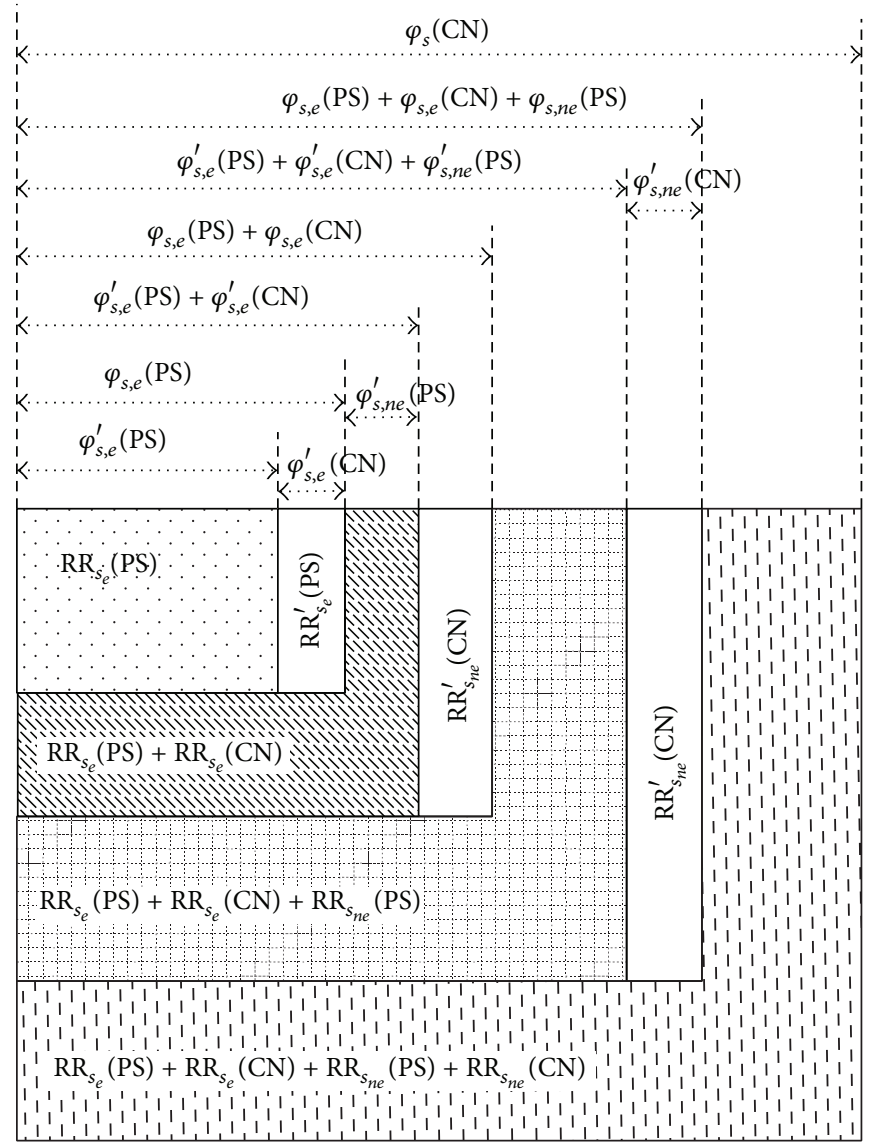

FIgURE 3: Frequency situation usage based CAMF.

priority bearer, and $\mathrm{cp}_{2}$ is the coefficient priority value of the lower priority bearer, both belonging to the same FCG.

The main objective of modeling a courteous frequency allocation constraints scheme is to guarantee a better QoS for the bearers of high priority and improve the performance of lower priority bearers.

In this model, all active bearers belonging to the same FCG group share the same radio resources according to the following conditions.

(i) All active bearers belonging to $\mathrm{FCG}_{n}$ may not use more than $\varphi_{n}\left(\mathrm{FCG}_{n}\right)$ radio resources.

(ii) All active bearers belonging to $\mathrm{FCG}_{n}$ and $\mathrm{FCG}_{n-1}$ cannot use more than $\varphi_{n-1}\left(\mathrm{FCG}_{n-1}\right)+\varphi_{n-1}^{\prime}\left(\mathrm{FCG}_{n-1}\right)$ resources;

(iii) And so forth.

(iv) All active bearers belonging to $\mathrm{FCG}_{n}+\mathrm{FCG}_{n-1}+$ $\cdots+\mathrm{FCG}_{1}$ have to use no more than $\varphi_{n-1}\left(\mathrm{FCG}_{n-1}\right)+$ $\varphi_{n-1}^{\prime}\left(\mathrm{FCG}_{n-1}\right)+\varphi_{n-2}\left(\mathrm{FCG}_{n-2}\right)+\varphi_{n-2}^{\prime}\left(\mathrm{FCG}_{n-2}\right)+\cdots+$ $\varphi_{1}\left(\mathrm{FCG}_{1}\right)+\varphi_{1}^{\prime}\left(\mathrm{FCG}_{1}\right)$.

(v) The sum of all frequency allocated resources should be less than the maximum quantity of radio resources that can be allocated, denoted by $M$. (vi) Each bearer within $\mathrm{FCG}_{k}, k \in\{1, C-1\}, C$ is the number of active FCG in the network, may allocate $\mathrm{RR}_{s_{k}}$ from $\varphi_{k}\left(\mathrm{FCG}_{k}\right)$ resources and $\mathrm{RR}_{s_{k+1}}^{\prime}$ from $\varphi_{k+1}\left(\mathrm{FCG}_{k+1}\right)$.

(vii) Higher priority bearers in $\mathrm{FCG}_{n}$ cannot allocate added resources by courteous scheme.

(viii) However, the sum of $\varphi_{k}\left(\mathrm{FCG}_{k}\right)$ for $k \in\{0, C-1\}$ can go beyond the threshold $M$

Given the conditions mentioned above, the mathematical modeling of G-CAMF model is given by the set of formulas (23).

The following joint forms represent the allocation constraints model for frequencies relevant to the adapted CAMF for $n$ FCG.

For each bearer in $\mathrm{FCG}_{k}, k \in\{1, C\}, C$ is the number of FCG groups. $C=n$ :

$$
\sum_{i=k}^{c} \mathrm{RR}_{s_{i}} \leq \sum_{i=k}^{c} \varphi_{i} \leq M
$$


(2) with the constraint

$$
\mathrm{RR}_{s_{C}}+\sum_{i=0}^{c-1}\left(\mathrm{RR}_{s_{i}}+\mathrm{RR}_{s_{i}}^{\prime}\right) \leq M .
$$

(3) For each bearer in $\mathrm{FCG}_{k}, i \in\{1, C-1\}, C=n$ :

$$
\mathrm{RR}_{s_{i}}^{\prime}=\mathrm{RR}_{s_{i}}+\mathrm{RR}_{s_{i+1}}^{\prime} .
$$

(4) For $C=n$,

$$
\mathrm{RR}_{s_{n}}^{\prime}=\mathrm{RR}_{s_{n}}
$$

(5) Finally, for $C=n$,

$$
\sum_{i=0}^{c} \varphi_{s, i} \geq M
$$

4.4. Radio Usage Situation Based Courteous Constraints Allocation Model for Frequencies: Application of the G-CAMF for Four Frequencies. Remember that the CAMF (courteous allocation model for frequencies) model is based on the CAM (courteous bandwidth constraints allocation model) [10]. CAMF, as it has been developed in this paper, manages access priority to the shared commercial frequency bands for PS and $\mathrm{CN}$ users without taking into account the urgency factor of the traffic. Although the PS traffic has a higher priority than $\mathrm{CN}$ traffic, but users within the same network, PS or CN are considered having the same priority level. Consequently, emergency calls will be treated in the same way than the less urgent calls. In order to effectively manage crisis situations, the information exchanged during times of disasters must be sent as a prior traffic. To meet this objective, CAMF is adapted to provide high priority traffic for traffic corresponding to crisis management.

Therefore, the aim of this new approach is to give a higher priority to the urgent traffic compared to another, especially, prioritize the urgent traffic $\mathrm{CN}$ compared to nonemergency traffic PS. On the other hand, the courteous process is redesigned so that the resources released for the PS urgent traffic type will be offered for urgent traffic $\mathrm{CN}$ traffic only. This will reduce the rate of loss of PS packets during the crisis management. However, nonemergency traffic of type $\mathrm{CN}$ will benefit only from nonemergency traffic PS radio resources offered by courtesy.

Figure 3 illustrates CAMF adaptation for shared commercial frequency allocation with constraints model for the emergency and nonemergency PS and CN traffic.

In this work, the mathematical formulation of the GCAMF is applied to the case of the allocation of radio resources to the PS and $\mathrm{CN}$ networks with supporting of the frequency situation usages, namely, emergency usage and nonemergency usage. This model is given as follows:

(i) frequency class groups (FCG) is set of bearers belonging to the same network, PS or $\mathrm{CN}$, and having priority coefficient belonging to the same values $\mathrm{cp}_{1}$ and $\mathrm{cp}_{2}$; (ii) bearers are mapped to FCG as follows:

(1) nonemergency $\mathrm{CN}$ bearers are mapped to $\mathrm{FCG}_{1}$;

(2) nonemergency PS bearers are mapped to $\mathrm{FCG}_{2}$;

(3) emergency $\mathrm{CN}$ bearers are mapped to $\mathrm{FCG}_{3}$;

(4) emergency PS bearers are mapped to $\mathrm{FCG}_{4}$.

Radio allocation and frequencies depend on the bearer network belonging, PS or CN, and the radio usage situation, emergency, or nonemergency. Therefore

(1) $\mathrm{CN}$ nonemergency situation is represented by the value 1 ;

(2) PS nonemergency situation is represented by the value 2 ;

(3) $\mathrm{CN}$ emergency situation is represented by the value 3;

(4) PS emergency situation is represented by the value 4 .

The following joint forms represent the allocation constraints model for frequencies relevant to the adapted CAMF for emergency or nonemergency situation usages.

(i) For each bearer in $\mathrm{FCG}_{k}, k \in\{1, C\}, C$ is the number of FCG groups. $C=4$ :

(1)

$$
\sum_{i=k}^{c} \mathrm{RR}_{s_{i}} \leq \sum_{i=k}^{c} \varphi_{i} \leq \varphi_{s}(\mathrm{CN})
$$

(2) with the constraint

$$
\mathrm{RR}_{s_{C}}+\sum_{i=0}^{c-1}\left(\mathrm{RR}_{s_{i}}+\mathrm{RR}_{s_{i}}^{\prime}\right) \leq \varphi_{s}(\mathrm{CN})
$$

(3) For each bearer in $\mathrm{FCG}_{k}, i \in\{1, C-1\}, C=n$,

$$
\mathrm{RR}_{s_{i}}^{\prime}=\mathrm{RR}_{s_{i}}+\mathrm{RR}_{s_{i+1}}^{\prime} .
$$

(4) For $C=n$,

$$
\mathrm{RR}_{s_{n}}^{\prime}=\mathrm{RR}_{s_{n}}
$$

(5) Finally, for $C=n$

$$
\sum_{i=0}^{c} \varphi_{s, i} \geq \varphi_{s}(\mathrm{CN})
$$

Note that CAMF, G-CAMF, and RUS-CAMF are the frequencies allocation constraints model adapted by CPA and $\mathrm{CPAwO}$ for the allocation of the radio resources. Note that the constraints are included in CPA and CPAwO algorithms to assure that the constraints are applied. 


\section{Simulation and Results}

In this section the most important results related to the simulation of $\mathrm{CPAwO}$ scheme are presented and commented on. The simulation is performed in Matlab. The different bearer type arrivals and finish times are generated randomly. PS traffic arrives 120 seconds after the arrival of the first commercial bearers. The four types of generated traffics are PS emergency, PS nonemergency, CN emergency, and $\mathrm{CN}$ nonemergency traffics. We simulate 2500 bearer arrivals for PS emergency, the same number for PS nonemergency, 8000 calls as CN emergency, and 3000 new bearers for $\mathrm{CN}$ nonemergency. The simulation duration is about 600 seconds.

Note that for all simulation graphs we consider three radio allocation schemes. The classical one [4], the courteous model that represents our previous contribution, namely, CPA model [4], and the "courteous + offload" model which represents the $\mathrm{CPAwO}$ algorithm modeled in this paper. Note that CPA model is an enhancement of the classical one by adapting the courteous scheme; it is based on CAMF model. Beside, CPAwO is based on RUS-CAMF and the classical model uses the constraints adapted in the paper [4].

Otherwise, remember that the $\mathrm{CPAwO}$ algorithm allocates resources within the LTE macrocell and LTE small cells. In this paper we use one macrocell and four small cells. The available radio resources quantities within the small cells are initiated randomly in the beginning of the simulation. The arrivals of bearer establishment requests to these small cells occur with respect to the offloading macrocells bearers and the bearers finish times within these cells are generated randomly.

Besides, we suppose that the LTE HetNet is used in this simulation in order to allow performing the D2D communications, although, we consider that $\mathrm{CPAwO}$ may not offload bearers if no resources are available in the small cells. In this case, $\mathrm{CPAwO}$ will have the same behaviour as $\mathrm{CPA}$ algorithm. Furthermore, both CPA and CPAwO cannot apply courteous scheme if the PSN resources are limited in the shared commercial radio. Finally, we suppose that the CN traffic is higher than the PSN traffic; in this case the $\mathrm{CN}$ traffic may have a critical level of QoS.

Figures 4 to 7 illustrate the number of active bearers in the network for the four bearer types simulated within this paper. The active bearers represent the bearers that are admitted in the network and still established within it. Remember that each bearer needs a quantity of radio resources to be admitted and active in the network. As number of active bearers for a kind of traffic increases, it will be allocated highest quantity of resources.

For the CPA results shown in Figures 4 and 5 depending on the PS traffic, the number of active bearers in the network is decreasing when courteous model is applied; comparing to the classical method, this is due to the courteous function applied by CPA algorithm. The aim of the courteous function is to release some PS radio resources from the higher priority traffic for the lower priority class traffic. Indeed, the number of commercial active bearers is more important when we

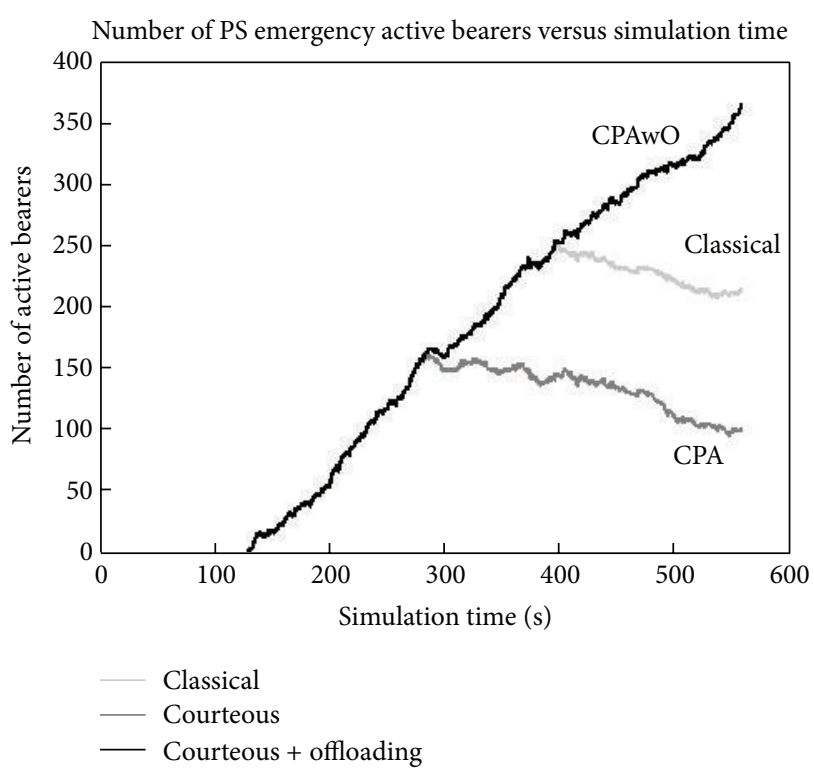

FIGURE 4: Number of PS emergency active bearers.

apply courteous scheme (CPA) than when we apply the classical method (Figures 6 and 7). This is possible only when the PS emergency traffic has a good QoS. On the other hand, we note an enhancement in the number of active bearers in the whole network when applying the $\mathrm{CPAwO}$ model, where the number of bearers which are active increased for both traffic types PS and CN and for the two situation usages emergency and nonemergency. Thus, the use of the public frequencies improves the QoS of all traffics types by increasing the number of the admitted bearers in the network.

Figures 4 to 7 show also that the three algorithms have the same behaviour before 300 seconds. This is due to congested or noncongested network. Hence, when resources are available, before 300 seconds, all bearers' requests are satisfied, but once the resources are limited, after 300 seconds, the network become congested and the lower priority bearers will get less resources comparing to the higher priority bearers. Note that the appearing of the congestion at 300 seconds depends especially on this simulation. The reasons of this are related to the number of arrivals and departures of bearers, which are generated randomly in these experiments.

On the other hand, the results show that CPA algorithm represented by the courteous scheme in the graph in Figure 8 enhances the QoS of the less priorities traffic by reducing the number of commercial blocking bearers. Furthermore, the $\mathrm{CPAwO}$ gets best results when Figure 8 shows that no bearers are blocked in the LTE HetNets network. This performance is reached thanks to the use of the small cells. The new arrived bearers of $\mathrm{CN}$ types are redirected to the small cells, so all bearers' establishment requests are accepted. Remember that the macrocell offloading is done transparently from the clients, so the use of CPAwO enhances the QoS of the commercial users without disturbing the clients.

In the same way, we see in Figures 9 and 10 that the number of blocked bearers with PS type is increased when 


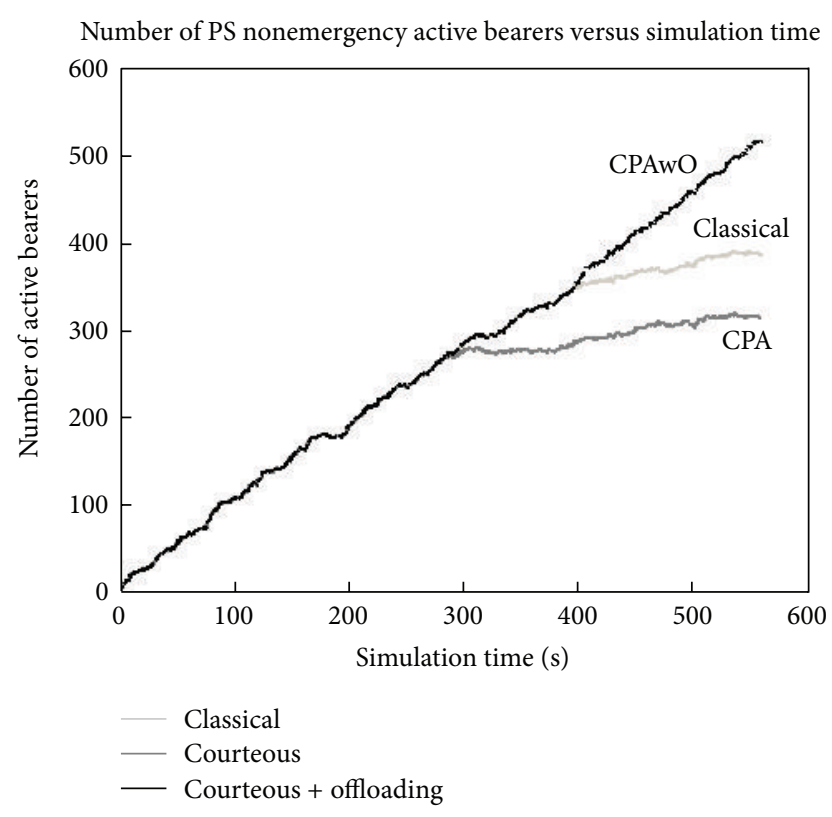

FIGURE 5: Number of PS nonemergency active bearers.

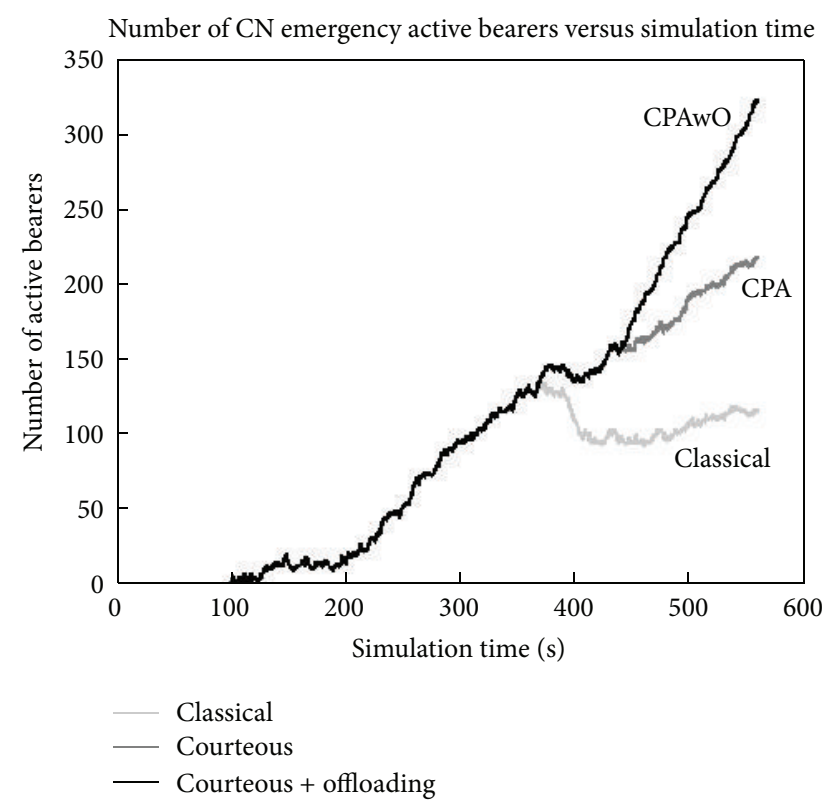

Figure 6: Number of CN emergency active bearers.

applying CPA algorithm. This is caused by the courteous mechanism which can give some radio resources to the lower priorities bearers as the number of blocked bearer does not reach the blocked bearer threshold. In the contrary, the results given by applying $\mathrm{CPAwO}$ illustrate that no PS bearer is blocked in the system for both emergency and nonemergency status usage. So, despite the use of the courteous mechanism in the CPAwO, the courteous bearers do not suffer from blocking phenomenon. Once again, this performance has
Number of CN nonemergency active bearers versus simulation time

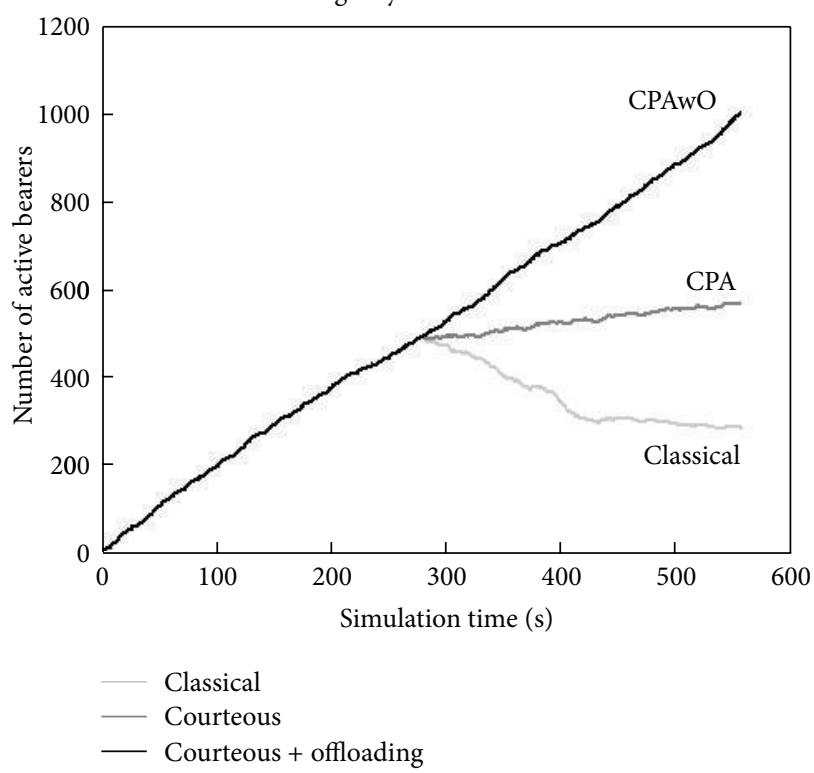

FIGURE 7: Number of CN nonemergency active bearers.

Number of $\mathrm{CN}$ nonemergency blocked bearers versus simulation time

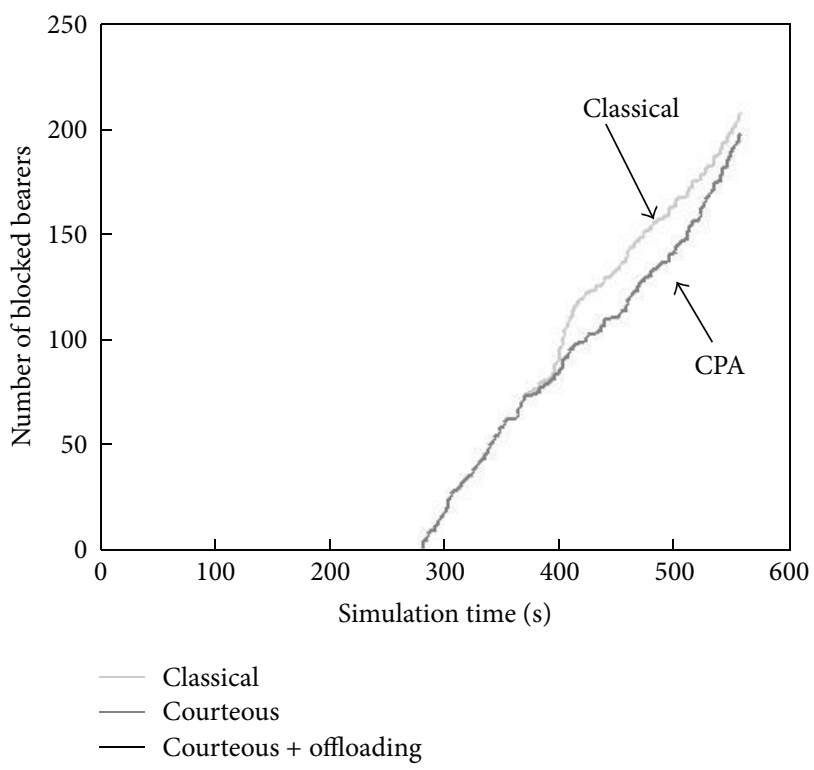

Figure 8: Number of CN nonemergency blocked bearers.

been reached thanks to the use of the small cells, which provide additional radio resources for the whole network.

Figure 11 represents the number of interrupted bearers in the network. Remember that $\mathrm{CPAwO}$ has active bearers in both macro- and small cells, so the preemption may occur in both cells if resources are limited. Note that for this simulation, we have preempted bearers only for the nonemergency commercial type bearers. The graphs in Figure 11 show that the preemption rate is reduced by the courteous mechanism, represented by the CPA algorithm. This happens thanks to the reallocation of the unused radio resources reserved to 
Number of PS emergency blocked bearers versus simulation time

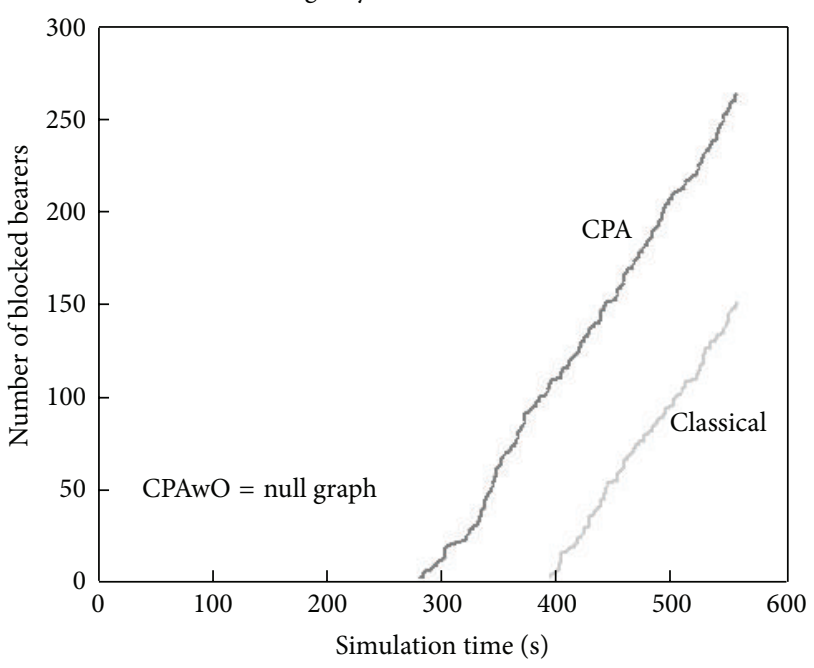

Classical

— Courteous

— Courteous + offloading

FIGURE 9: Number of PS emergency blocked bearers.

Number of PS nonemergency blocked bearers versus simulation time

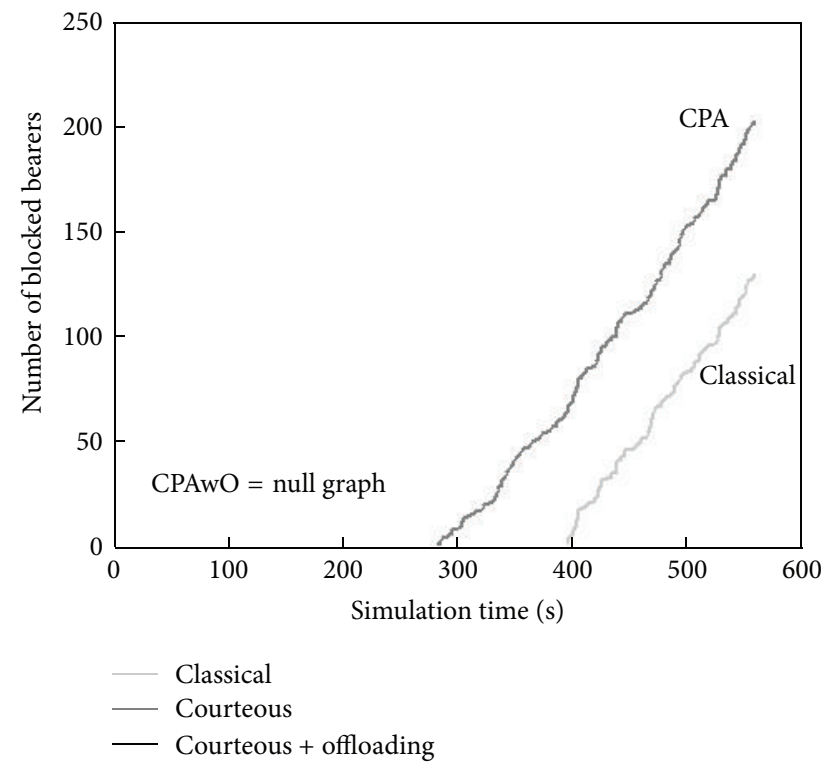

FIGURE 10: Number of PS nonemergency blocked bearers.

the higher priorities traffic in order to be used by the low priorities traffic. In this way the allocation model is enhanced by avoiding the underuse of frequencies bands. Better yet, the CPAwO eliminates outright the preemption within the LTE HetNets network. Remember that for CPAwO when a new bearer arrive to the macrocell and it cannot be admitted because of the lack of resources, it will not interrupt another bearer even if it is more prioritized than the active one. It will rather be redirected to the small cell. The preemption does not occur until the resources are limited in the whole network.

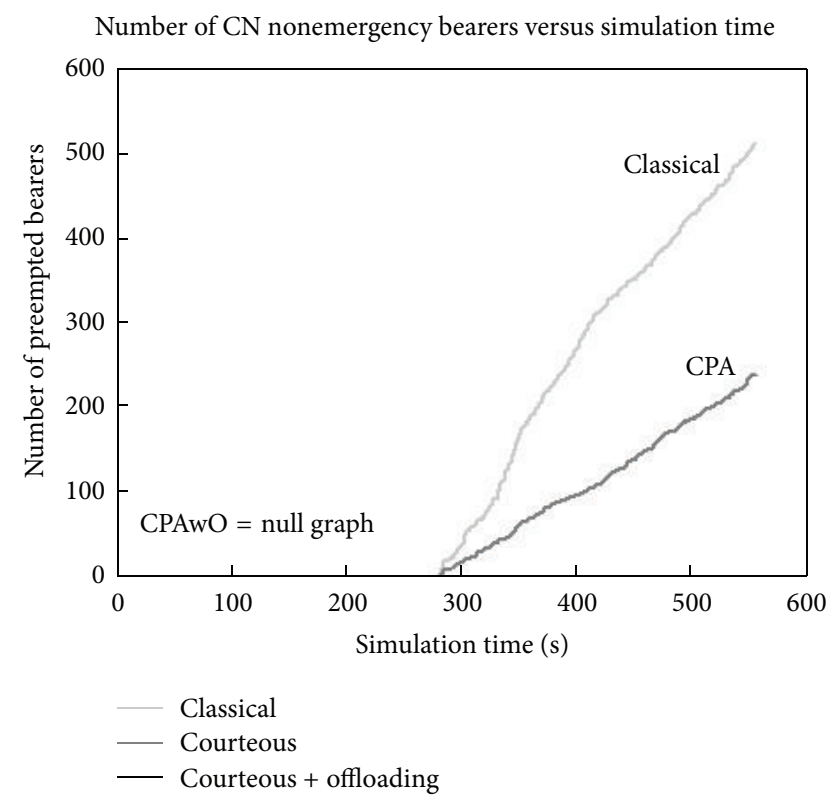

FIGURE 11: Number of CN nonemergency preempted bearers.

This technique makes the system more stable by reducing the number of interrupted bearers.

\section{Conclusion}

This paper investigates the priority access to the shared commercial radio for the public safety over the LTE heterogeneous networks. A new approach for Courteous Priority Access to the shared commercial radio with offloading (CPAwO) for the PSN over LTE HetNets networks is presented. This scheme manages the radio resources in an LTE HetNets network for assuring and enhancing the PS user's access to the commercial shared radio over this network. The congestion issue occurring in the LT macrocell is addressed by the offloading mechanism "HOW" provided by $\mathrm{CPAwO}$ protocol. On the other hand, three frequency allocation constraints models are modeled in this paper by, namely, the CAMF, the RUS-CAMF, and the G-CAMF models. CPA and $\mathrm{CPAwO}$ use the frequency allocation constraints models to manage the resources. CAMF detailed the frequency allocation scheme for two traffic types, especially the PS traffic and the CN one. As CAMF Model, RUS-CAMF model considers two types of traffics, namely Public Safety traffic and Commercial traffic. In addition, RUS-CAMF considers the two situations usages of traffic, emergency and nonemergency. The third model, G-CAMF, is a generalization of the CAMF for $\mathrm{n}$ traffic classes and situations.

The simulation results of the CPA and CPAwO mechanisms show that while the QoS of the higher priority traffic is acceptable CPA algorithm enhances the QoS of the lower priorities traffic by admitting more bearers belonging to theme. On the other hand, CPAwO scheme corrects shortcomings of CPA while providing the same benefits and even better for the all types of traffics in any usages situation. 


\section{Future Works}

To improve the performance of $\mathrm{D} 2 \mathrm{D}$ communications, we have developed several algorithms. Two algorithms of signalling have been modeled, namely, RBC (reliable butterfly construction) algorithm [21] and LBS-AOMDV (load balancing AOMDV) algorithm [22]. Both of them define a set of routes connecting a source to a destination. RBC builds several butterfly effects in a WMN and ad hoc networks. This allows using network coding mechanism for D2D transmissions. RBC allows also the routes backup after a butterfly failure. If RBC constructs no butterfly effect, then the LBSAOMDV algorithm will be applied to get a multipath within the WMN. Constructing several paths is very pertinent to the D2D communication since it ensures the dispatch operations, an essential mechanism for the first responders to exchange data during situations of crisis. The security aspect has not been neglected in our study. The G-SNCDS (generalized secure network coding based data splitting) algorithm, an extension of our SNCDS algorithm [23], will be developed for the transmission of data based on the splitting technique, in order to address the confidentiality, the integrity, and the availability attacks in the WLAN networks.

Furthermore, some studies are dealing with the different cells cooperation to enhance the resources management, the power allocation, and the QoS in the HetNets networks [2426]. Therefore, to improve the resources management for the PSN network, base stations of different transmissions (macrocells, microcells, picocells, and small cells) will be considered in our future studies to enhance the offloading mechanism. New schemes will be developed in order to delay the preemption of PSN bearers during the situation of crisis when the resources are limited in the WLAN network.

Finally, a set of experiments will be carried out in order to show the performance of CPA and CPAwO in terms of end-to-end QoS (latency, packet loss, and throughput) and congestions.

\section{Definition of Used Variables}

$\varphi_{s}(\mathrm{PS}): \quad$ PS shared radio resources constraint

$\varphi_{s}(\mathrm{CN}): \quad \mathrm{CN}$ shared radio resources constraint

$\varphi_{s, e}(\mathrm{PS}): \quad$ PS shared radio resources constraint for emergency situations (ES)

$\varphi_{s, e}(\mathrm{CN}): \mathrm{CN}$ shared radio resources constraint for ES

$\varphi_{s, n e}(\mathrm{PS}): \quad$ PS shared radio resources constraint for nonemergency situations (NES)

$\varphi_{s, n e}(\mathrm{CN}): \mathrm{CN}$ shared radio resources constraint for NES

$\varphi_{s, e}^{\prime}(\mathrm{CN}): \mathrm{CN}$ shared radio resources constraint for ES, got by courteous scheme

$\varphi_{s, e}^{\prime}(\mathrm{PS}): \quad$ PS shared radio resources constraint for ES given by courteous to $\mathrm{CN}$

$\varphi_{s, \text { ne }}^{\prime}(\mathrm{CN}): \mathrm{CN}$ shared radio resources constraint for NES got by courteous scheme

$\varphi_{s, \text { ne }}^{\prime}(\mathrm{PS}):$ PS shared radio resources constraint for non-NES, given by courteous to $\mathrm{CN}$

$\mathrm{RR}_{s}(\mathrm{PS}): \quad \mathrm{PS}$ allocated radio resources
$\mathrm{RR}_{s}(\mathrm{CN}): \quad \mathrm{CN}$ allocated radio resources

$\mathrm{RR}_{s, e}(\mathrm{PS}): \quad$ PS allocated radio for ES

$\mathrm{RR}_{s, e}(\mathrm{CN}): \quad \mathrm{CN}$ allocated radio for $\mathrm{ES}$

$\mathrm{RR}_{s, \text { ne }}(\mathrm{PS})$ : $\quad$ PS allocated radio for NES

$\mathrm{RR}_{s, \text { ne }}(\mathrm{CN}): \mathrm{CN}$ allocated radio for NES

$\mathrm{RR}_{s, e}^{\prime}(\mathrm{CN})$ : CN allocated radio for ES, among the PS shared frequencies, after the courteous process

$\mathrm{RR}_{s, \mathrm{ne}}^{\prime}(\mathrm{PS}):$ PS allocated radio for NES, among the PS and $\mathrm{CN}$ shared frequencies for ES, after courteous process

$\mathrm{RR}_{s, \mathrm{ne}}^{\prime}(\mathrm{CN})$ : $\mathrm{CN}$ allocated radio for NES, among the other shared frequencies after the courteous process

M: $\quad$ Maximum reservable radio resources. $M=\varphi_{s}(\mathrm{CN})$

$\varphi_{\text {Max }}: \quad$ Maximum amount of radio resources

$\varphi_{d}(\mathrm{PS}): \quad$ PS dedicated radio resources constraint

$\varphi_{s}(\mathrm{PS}): \quad$ PS shared radio resources constraint

$\varphi_{d}(\mathrm{CN}): \quad \mathrm{CN}$ dedicated radio resources

$\varphi_{s}(\mathrm{CN}): \quad \mathrm{CN}$ shared radio resources constraint

$\varphi_{s}^{\prime}(\mathrm{CN}): \quad \mathrm{CN}$ shared radio resources constraint got by courteous scheme

$\varphi_{s}^{\prime}(\mathrm{PS}): \quad$ PS shared radio resources constraint given by courteous to $\mathrm{CN}$

$\mathrm{RR}_{d}(\mathrm{PS}): \quad \mathrm{PS}$ dedicated radio resource

$\mathrm{RR}_{d}(\mathrm{CN}): \quad$ PS allocated radio among the PS dedicated frequencies

$\mathrm{RR}_{s}(\mathrm{PS}): \quad \mathrm{PS}$ allocated radio among the PS shared frequencies

$\mathrm{RR}_{s}(\mathrm{CN}): \quad \mathrm{CN}$ allocated radio among the PS dedicated frequencies

$\mathrm{RR}_{s}^{\prime}(\mathrm{PS})$ : $\quad$ PS allocated radio among the PS shared frequencies after courteous process

$\mathrm{RR}_{s}^{\prime}(\mathrm{CN})$ : $\quad$ PS allocated radio among the PS shared frequencies after the courteous process

$\mathrm{R}_{\mathrm{th}, \mathrm{s}}(\mathrm{PS})$ : Courteous threshold.

\section{Conflict of Interests}

The authors declare that there is no conflict of interests regarding the publication of this paper.

\section{References}

[1] D. Astely, E. Dahlman, G. Fodor, S. Parkvall, and J. Sachs, "LTE release 12 and beyond," IEEE Communications Magazine, vol. 51, no. 7, pp. 154-160, 2013.

[2] R. Q. Hu and Y. Qian, Heterogeneous Cellular Networks, John Wiley \& Sons, New York, NY, USA, 2013.

[3] T. Doumi, M. F. Dolan, S. Tatesh et al., "LTE for public safety networks," IEEE Communications Magazine, vol. 51, no. 2, pp. 106-112, 2013.

[4] S. Borkar, D. Roberson, and K. Zdunek, "Priority Access for public safety on shared commercial LTE networks," in Proceedings of the Technical Symposium at ITU Telecom World (ITU WT '11), pp. 105-110, Geneva, Switzerland, October 2011.

[5] C. Tata and M. Kadoch, "Courteous Allocation Model for Frequencies for Public Safety Priority Access to the Shared 
Commercial Radio in LTE Heterogeneous network," presented at the FiCloud, Barcelona, Spain (Submitted), 2014.

[6] H. Ryan and J. M. Peha, "Enabling public safety priority use of commercial wireless networks," Homeland Security Affairs, vol. 9, article 13, 2013.

[7] R. Blom, P. D. Bruin, J. Eman et al., "Public safety communication using commercial cellular technology," in Proceedings of the 2nd International Conference on Next Generation Mobile Applications, Services, and Technologies (NGMAST '08), pp. 291296, Cardiff, UK, September 2008.

[8] X. Lin, J. G. Andrews, A. Ghosh, and R. Ratasuk, "An overview on 3GPP device-to-device proximity services," CoRR abs/1310.0116, 2013.

[9] B. Raghothaman, E. Deng, R. Pragada, G. Sternberg, T. Deng, and K. Vanganuru, "Architecture and protocols for LTE-based device to device communication," in Proceedings of the International Conference on Computing, Networking and Communications (ICNC '13), pp. 895-899, San Diego, Calif, USA, January 2013.

[10] C. Tata and M. Kadoch, "CAM: courteous bandwidth constraints allocation model," in Proceedings of the 20th International Conference on Telecommunications (ICT '13), pp. 1-5, May 2013.

[11] Le Faucheur and W. Lai, "Maximum Allocation Bandwidth Constraints Model for Diffserv-aware MPLS Traffic Engineering," IETF, RFC 4125, June 2005.

[12] F. Le Faucheur, "Russian dolls bandwidth constraints model for Diffserv-aware MPLS traffic engineering," IETF, RFC 4127, 2005.

[13] M. Qian, Y. Huang, J. Shi, Y. Yuan, L. Tian, and E. Dutkiewicz, "A novel radio admission control scheme for multiclass services in LTE systems," in Proceedings of the IEEE Global Telecommunications Conference (GLOBECOM '09), pp. 1-6, IEEE, Honolulu, Hawaii, USA, December 2009.

[14] R. Kwan, R. Arnott, R. Trivisonno, and M. Kubota, "On preemption and congestion control for LTE systems," in Proceedings of the IEEE 72nd Vehicular Technology Conference Fall (VTC2010-Fall '10), September 2010.

[15] S. Chadchan and C. Akki, "Priority-scaled preemption of radio resources for 3GPP LTE networks," International Journal of Computer Theory and Engineering, vol. 3, no. 6, 2011.

[16] A. Pyattaev, K. Johnsson, S. Andreev, and Y. Koucheryavy, "3GPP LTE traffic offloading onto WiFi Direct," in Proceedings of the IEEE Wireless Communications and Networking Conference Workshops (WCNCW '13), pp. 135-140, Shanghai, China, April 2013.

[17] W. Yoon and B. Jang, "Enhanced non-seamless offload for LTE and WLAN networks," IEEE Communications Letters, vol. 17, no. 10, pp. 1960-1963, 2013.

[18] H. Ishii, X. Cheng, S. Mukherjee, and B. Yu, "An LTE offload solution using small cells with D2D links," in Proceedings of the IEEE International Conference on Communications Workshops (ICC '13), pp. 1155-1160, June 2013.

[19] L. Hu, L. L. Sanchez, M. Maternia et al., "Modeling of WiFi IEEE 802.11ac offloading performance for 1000x capacity expansion of LTE-advanced," in Proceedings of the 78th IEEE Vehicular Technology Conference (VTC Fall '13), pp. 1-6, Las Vegas, Nev, USA, September 2013.

[20] M. Kadoch and C. Tata, "Courteous algorithm: performance optimization in WiMAX networks," in Proceedings of the 4th International Conference on Communications and Information Technology (CIT '10), pp. 23-32, July 2010.
[21] C. Tata and M. Kadoch, "RBC: reliable butterfly network construction algorithm for network coding in wireless mesh network," in Proceedings of the 13th WSEAS International Conference on Applied Informatics and Communications (AIC '13), August 2013.

[22] C. Tata and M. Kadoch, "Multipath routing algorithm for device-to-device communications for public safety over LTE heterogeneous networks," in Proceedings of the 1st International Conference on Information and Communication Technologies for Disaster Management (ICT-DM '14), Algiers, Algeria, 2014.

[23] C. Tata and M. Kadoch, "Secure network coding based data splitting for public safety D2D communications over LTE heterogeneous networks," in 14th IEEE International Conference on Computer and Information Technology (CIT '14), Tenerife, Spain, 2014.

[24] R. Q. Hu and Y. Qian, "An energy efficient and spectrum efficient wireless heterogeneous network framework for $5 \mathrm{G}$ systems," IEEE Communications Magazine, vol. 52, no. 5, pp. 94101,2014

[25] Q. C. Li, R. Q. Hu, Y. Xu, and Y. Qian, "Optimal fractional frequency reuse and power control in the heterogeneous wireless networks," IEEE Transactions on Wireless Communications, vol. 12, no. 6, pp. 2658-2668, 2013.

[26] Q. Li, R. Q. Hu, Y. Qian, and G. Wu, "Intracell cooperation and resource allocation in a heterogeneous network with relays," IEEE Transactions on Vehicular Technology, vol. 62, no. 4, pp. 1770-1784, 2013. 

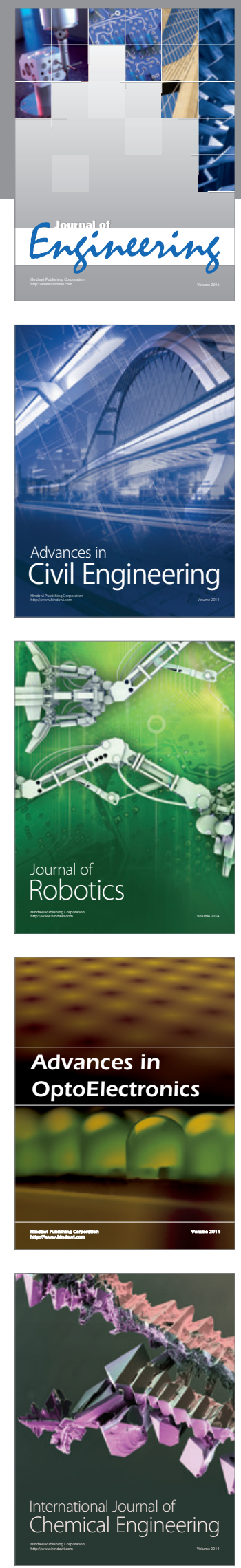

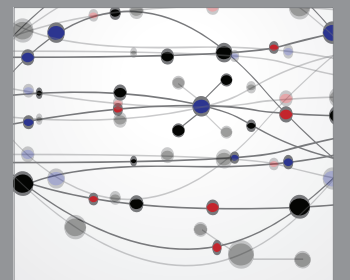

The Scientific World Journal
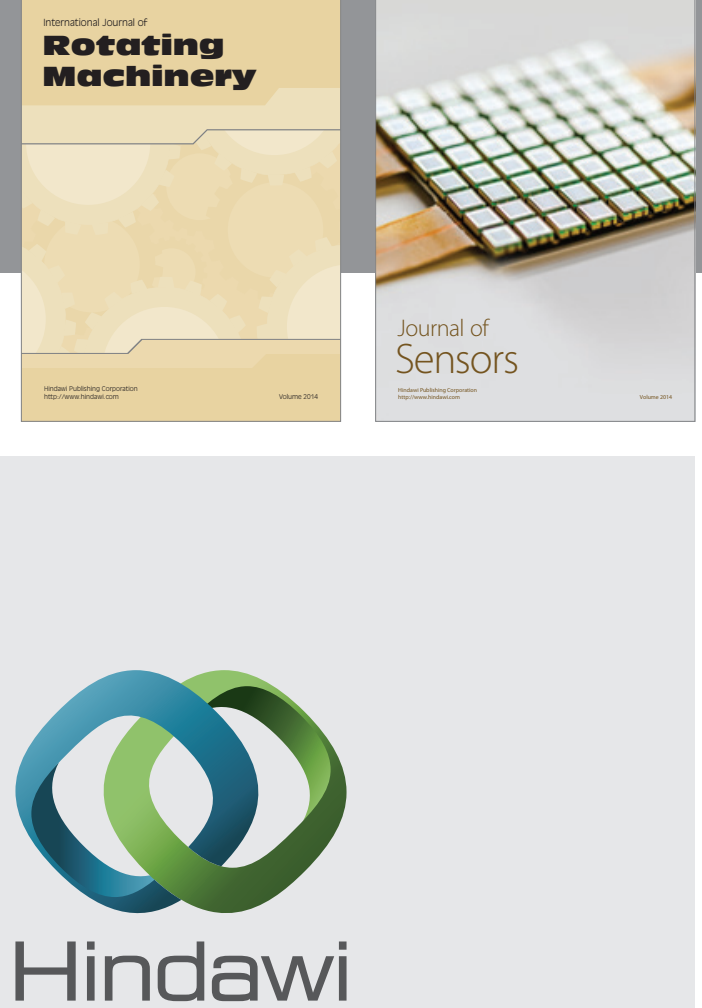

Submit your manuscripts at http://www.hindawi.com
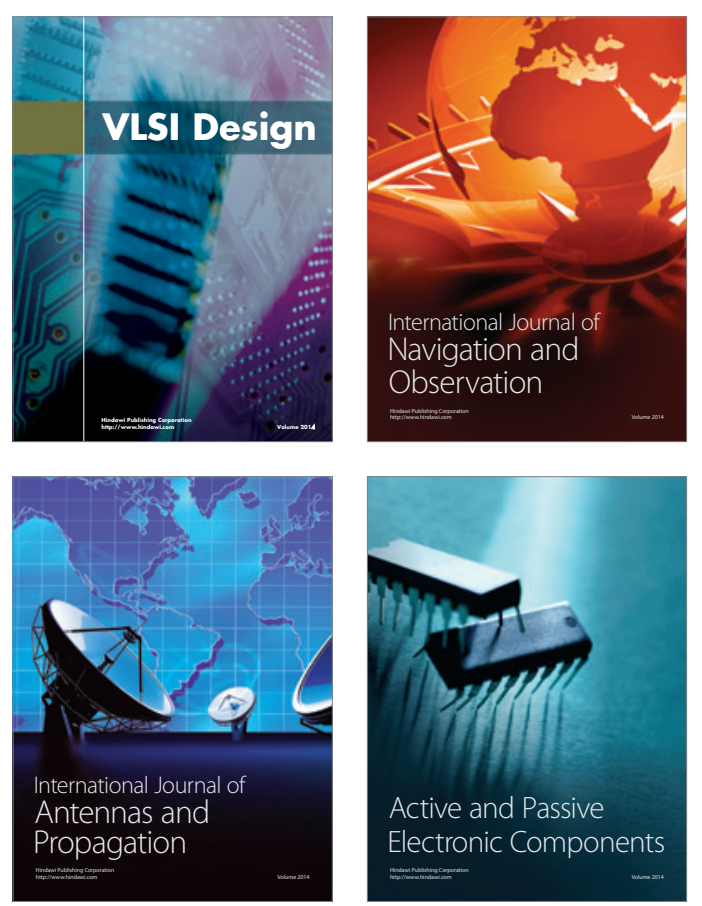
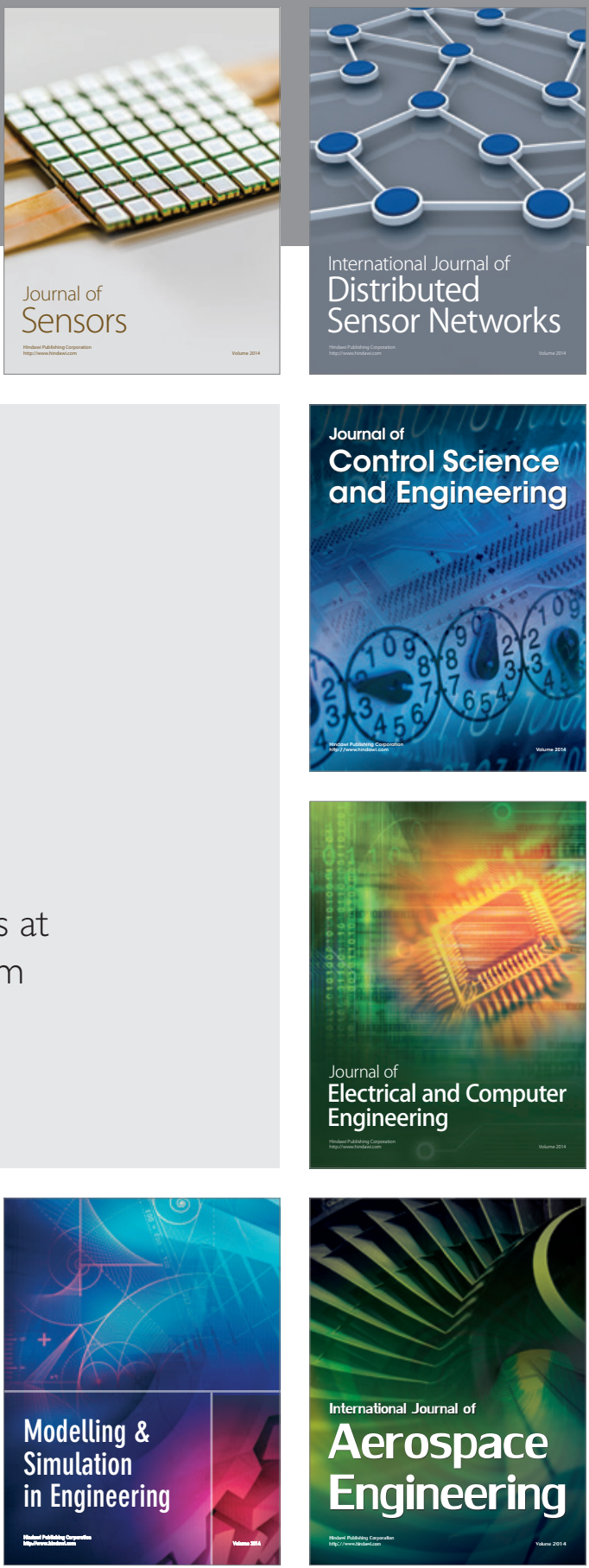

Journal of

Control Science

and Engineering
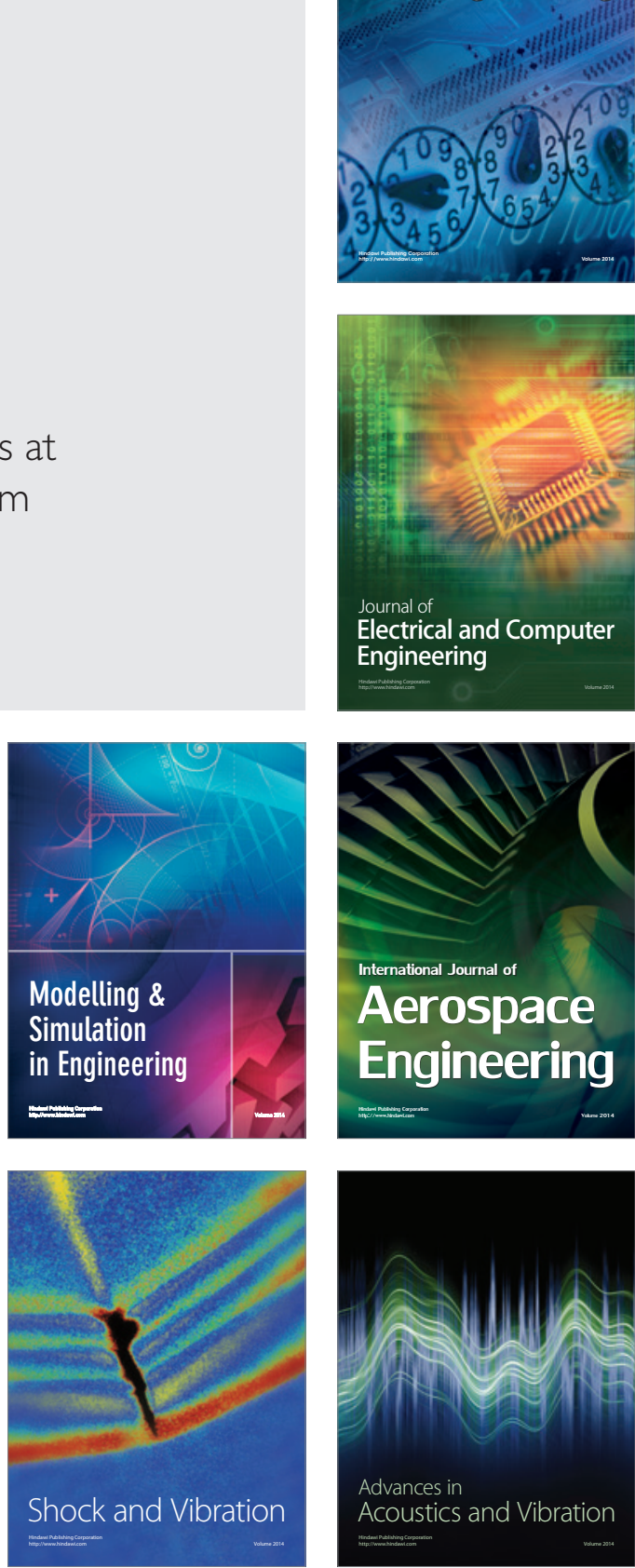